\title{
Behaviour of biomass particles in a large scale (2-4MWth) bubbling bed reactor
}

\author{
T. Berdugo Vilches, E. Sette \& H. Thunman \\ Department of Energy and Environment, \\ Chalmers University of Technology, Sweden
}

\begin{abstract}
Biomass is regarded as an interesting fuel for energy-related processes owing to its renewable nature. However, the high volatile content of biomass adds a number of difficulties to the fuel conversion and process operation. In the context of fluidized bed reactors, several authors have observed that devolatilizing fuel particles tend to float on the surface of a gas-fluidized bed of finer solids. This behaviour, known as segregation, leads to undesired effects such as poor contact between volatiles and bed material. Previous investigations on segregation of gas-emitting particles in fluidized beds are conducted in small units and they are often operated at rather low gas velocities, typically between the minimum fluidization velocity (umf) and $2 \cdot \mathrm{umf}$. Therefore, it is not known to what extent such results are of relevance for industrial scale units and for higher fluidization velocities that are commonly used in large bubbling beds. In this paper the behaviour of biomass particles in a large scale bubbling bed reactor is investigated. Tests were conducted at a wide range of fluidization velocities with three different bed materials of varying particle size and density. The fuel was wood pellets and the fluidization medium was steam, which makes the findings relevant for indirect gasification, chemical looping combustion (CLC) and bubbling bed combustion applications. The experiments were recorded by means of a digital video camera and the digital images were subsequently analysed qualitatively. The results show high level of segregation at fluidization velocity up to 3.5 umf. Beyond this point fuel mixing was significantly enhanced by increasing fluidization velocities. At the highest fluidization velocity tested (i.e. $>8$ umf), a maximum degree of mixing was achieved.

Keywords: bubbling bed, biomass, fuel segregation, digital image analysis.
\end{abstract}




\section{Introduction}

Mixing of solid fuel particles in a gas fluidized bed is a relevant problem to a number of fuel conversion technologies. Fuel mixing refers to mixing of fuel particles with bed material and/or fuel particles with fluidizing gas, in vertical and horizontal directions. Mixing of fuel and bed material particles in vertical direction is crucial when reactions between fuel and bed material are desired, e.g. for catalytic gasification and chemical looping combustion (CLC). In these examples, part of the fuel conversion takes place when the volatile matter released by the fuel reacts with the bed particles. If the fuel does not mix with the bed, volatile species may leave the reactor without interacting with the bed, thereby decreasing the performance of the reactor.

Lack of axial mixing, commonly known as segregation, originates from differences in properties of the fluidized particles, mainly particle size and density [1]. This phenomenon has been widely investigated for binary systems, for which Rowe et al. used the terms 'flotsam' and 'jetsam' to refer to the particles that tend to float and sink respectively [2]. In solid fuel conversion in fluidized beds, the fuel particles behave as 'flotsam' and tend to accumulate close to the surface of the bed, while the bed material behave as 'jetsam' and tend to settle to the bottom . The degree of fuel segregation is closely dependent on fluidization velocity. Experience from cold flow models with binary mixtures of sand and light biomass particles have shown that segregation is strong at fluidization velocities close to minimum fluidization $\left(\mathrm{u}_{\mathrm{mf}}\right)$, whereas fuel mixing takes place as fluidization velocity increases [3].

The 'flotsam' behaviour of the lighter fuel particles in a fluidized bed is accentuated in fuels with high volatile content, such as biomass and low-rank coal. The release of volatiles in hot conditions results in the formation of bubbles, which tend to lift the fuel particles to the surface of the bed [4-6]. These bubbles are commonly known as endogenous bubbles to differentiate them from those generated by the fluidization gas, which are denominated exogenous bubbles. Previous research on segregation of gas emitting particles has focused on quantifying the lifting time and/or lifting force due to the formation of endogenous bubbles [4-7]. The experiments were performed in small reactors with a single fuel particle and at incipient fluidization velocity [4-7] or fluidization velocities up to $2 \cdot \mathrm{u}_{\mathrm{mf}}$ [7].

The operating conditions in industrial scale energy conversion units differ considerably from those at which previous research on segregation was conducted. In real applications, multiple devolatilizing fuel particles are present in the reactor, which interact with numerous endogenous and exogenous bubbles. The complexity of the mixing situation in large scale renders unclear to which extent existing results can be extrapolated to industrial size bubbling bed reactors.

In this work, the segregation behaviour of devolatilizing biomass particles is investigated experimentally in a large bubbling bed reactor. The aim of the present work is to increase understanding of the segregation phenomena and how it is affected by the fluidization velocity and the bed material properties. For this 
purpose, a method to compare the level of segregation of the fuel particles in a bubbling bed is developed. An indicator for the degree of segregation is proposed, which consists of the fraction of the bed surface that is covered by the fuel particles. In order to quantify this indicator, the bed surface is video recorded, and the images are subsequently analysed by digital image analysis (DIA) techniques.

\section{Experimental}

\subsection{Experimental apparatus}

The experiments were conducted in the dual fluidized bed gasifier at Chalmers University of Technology. The Chalmers system consists of a $12 \mathrm{MW}_{\text {th }}$ circulating fluidized bed boiler (CFB) coupled to a 2-4MWth bubbling bed gasifier. The bed material circulates between the two reactors, thereby transferring heat from the combustor to the gasifier. In this study, the reactor of interest is the gasifier, which is briefly described below. For further details about the Chalmers system the reader is referred to [8-10].

The gasifier has a cross-section of $1.44 \mathrm{~m}^{2}$, and the bed material flows into and out of the reactor via the inlet and outlet loop seals, respectively. The fluidization agent is steam, and the fuel is fed by gravity onto the surface of the bed through a fuel chute. The gasifier operates at atmospheric pressure and at approximately $820^{\circ} \mathrm{C}$. Figure 1(a) provides a schematic drawing of the interior of the gasification reactor, where the location of the fuel feeding point, bed material inlet and outlet are indicated. A view of the front wall of the reactor is shown in Figure 1(b). The fuel chute and the gas exit are indicated by the arrows on the right and left hand side of the figure, respectively. The third arrow at the centre of the picture points at the port where the camera probe is mounted, which has an angle of $45^{\circ}$ with respect to the bed surface.

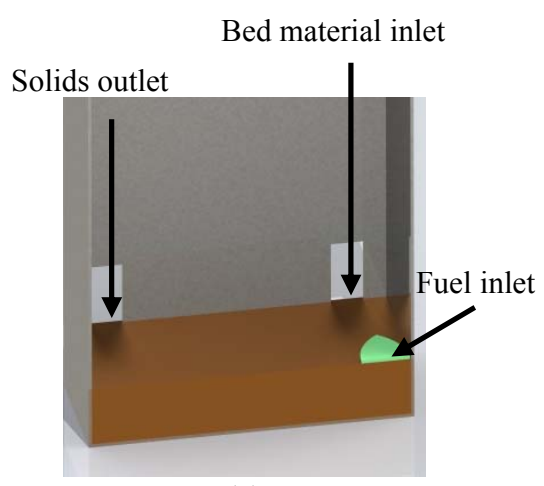

(a)

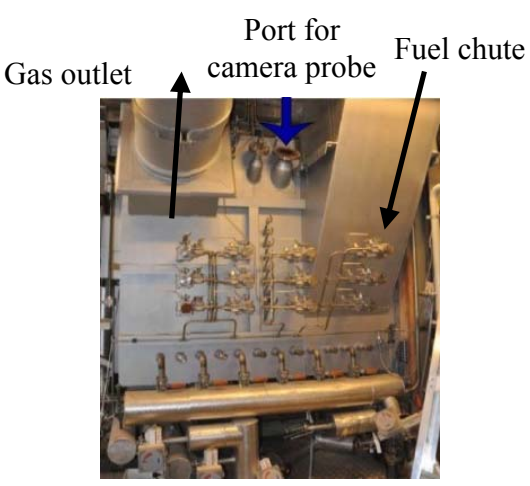

(b)

Figure 1: Chalmers gasifier: (a) schematic drawing of the interior of the reactor, front wall removed; (b) view of the front wall. 
In the present work, two different cameras were used, and a summary of their characteristics can be found in Table 1. The first camera records colour videos at a very high resolution and it does not have the possibility to mount additional external lenses. The second camera records greyscale videos at a slightly lower resolution and it has the possibility to mount an external temperature resistant lens, which increases the robustness of the measurement system in the harsh environment of the gasifier.

The same probe was used for both cameras; however, the location of the camera inside the probe differs as shown in Figure 2. The probe is equipped with a cooling jacket, which allows for a flow of water that keeps the temperature in the compartment where the camera is at $25^{\circ} \mathrm{C}$. Additionally, the probe is purged with nitrogen gas in order to avoid condensation of tars and keep the sand particles away from the glass.

Table 1: Summary of camera characteristics.

\begin{tabular}{|ccccc|}
\hline Manufacturer & Model & Dimensions (mm) & Resolution & Colour \\
\hline Sony & $\begin{array}{c}\text { Ipela SNC- } \\
\text { CH } 210\end{array}$ & $\begin{array}{c}\text { Diameter:44 } \\
\text { Length: } 93\end{array}$ & $1280 \times 720$ & Yes \\
\hline Unibrain & $630 \mathrm{~b}$ & $\begin{array}{c}\text { Length: } 38 \\
\text { Width: } 29 \\
\text { Height: } 29\end{array}$ & $1024 \times 768$ & No \\
\hline
\end{tabular}

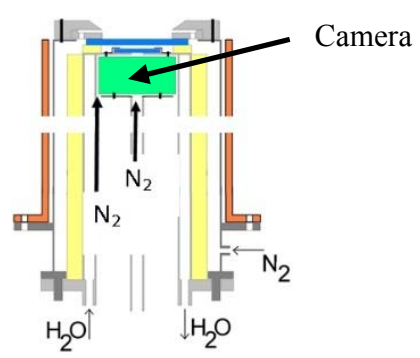

(a)

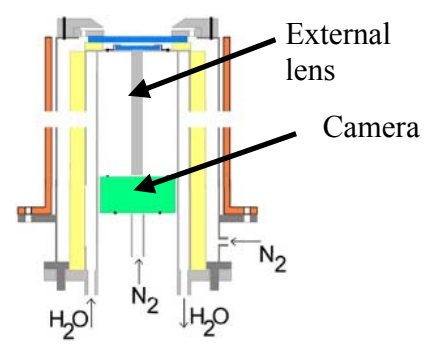

(b)

Figure 2: Camera probe (a) with the first camera (Sony Ipela SNC-CH 210) used in the experiments; (b) with the second camera (Unibrain 630).

\subsection{Experimental procedure}

Pelletized wood is fed continuously to the reactor at a rate of $200 \mathrm{~kg}_{\text {fuel }} / \mathrm{h}$. It is important to run all tests at the same fuel flow to enable comparison of the fraction of bed covered by the fuel in the different cases. The pellets have a diameter of $6 \mathrm{~mm}$ and have irregular length ranging from 10 to $20 \mathrm{~mm}$. The density of the wood pellets is approximately $1190 \mathrm{~kg} / \mathrm{m}^{3}$. The typical volatile content of this fuel is $80 \%$ on mass (dry basis), and the moisture is below $10 \%$ (w/w\%). 
Three different bed materials were investigated: two quartz sands with different mean particle sizes, and one sand-like material of higher density. The three materials were supplied by Sibelco and they are, hereby, referred to as coarse sand, fine sand and heavy sand. The mean particle size and density of the three bed materials are summarized on Table 2 .

Table 2: Mean particle size of the three bed material tested.

\begin{tabular}{|cll|}
\hline Fine sand & $244 \mu \mathrm{m}$ & $2600 \mathrm{~kg} / \mathrm{m}^{3}$ \\
\hline Coarse sand & $320 \mu \mathrm{m}$ & $2600 \mathrm{~kg} / \mathrm{m}^{3}$ \\
\hline Heavy sand & $180 \mu \mathrm{m}$ & $4200 \mathrm{~kg} / \mathrm{m}^{3}$ \\
\hline
\end{tabular}

The flow of steam is changed stepwise to alter the gas velocity $\left(\mathrm{u}_{\mathrm{o}}\right)$, thereby changing the fluidization behaviour of the bed. At least three different velocities are tested for each material, and a video of approximately 2 minutes is recorded for each case when stable steam flow is achieved. In order to compare the results, two different fluidization parameters are used, which are $\left(\mathrm{u}_{0} / \mathrm{u}_{\mathrm{mf}}\right)$ and $\left(\mathrm{u}_{0}-\mathrm{u}_{\mathrm{mf}}\right)$. These parameters are known as the fluidization number and excess gas velocity, respectively, and they are commonly used in fluidization literature to characterize fluidization. The minimum fluidization velocity of the bed particles are calculated for the mean particle size and the operating conditions according to the empirical correlations by Aerov and Todes [11]. A summary of the experimental matrix can be found on Table 3 .

Table 3: Experimental matrix.

\begin{tabular}{|lccc|}
\hline Bed material type & $\begin{array}{c}\text { Steam velocity, } \\
\mathbf{u}_{\mathbf{0}}(\mathbf{m} / \mathbf{s})\end{array}$ & $\mathbf{u}_{\mathbf{0}} / \mathbf{u}_{\mathbf{m f}}$ & $\left(\mathbf{u}_{\mathbf{0}}-\mathbf{u}_{\mathbf{m f}}\right)$ \\
\hline Fine sand & $0.12 / 0.13 / 0.15$ & $4.4 / 5.1 / 5.9$ & $0.09 / 0.11 / 0.13$ \\
\hline Coarse sand & $0.12 / 0.15 / 0.20$ & $2.6 / 3.5 / 4.6$ & $0.07 / 0.11 / 0.16$ \\
\hline \multirow{2}{*}{ Heavy sand } & $0.09 / 0.13 / 0.15 /$ & $4.1 / 5.7 / 6.5 /$ & $0.07 / 0.11 / 0.13 /$ \\
& $0.18 / 0.19$ & $7.7 / 8.1$ & $0.16 / 0.17$ \\
\hline
\end{tabular}

\section{Data processing}

The recorded videos are processed offline with a standard desktop computer using a computer vision system based on the ideas given by Bradski and Kaehler [12]. Since two cameras are used, preparation of the untreated videos is required to enable comparable evaluation of the frames. Therefore, the videos analysis is performed in greyscale and for the same region of interest (ROI), i.e. same area of the bed is analysed. The ROI is selected to be the central part of the bed and only the region containing the surface of the bed, thus discarding the visible walls of the reactor.

The determination of the bed area covered with fuel particles is done by segmentation of the fuel particles from each frame. The fuel particles are larger in size and colder than the bulk solids and, thus, they appear as dark regions in 
the video frames. Since the fuel particles have similar size, a Laplacian of Gaussian (LoG) [13] filter can be used to detect the particles. By applying the LoG filter, dark regions (of a size within the predefined filtering range) yields a strong negative response and the remaining part of the image yields a response around zero. By thresholding the filter response for negative values the fuel particles are successfully segmented from the background. The treated images are subsequently used for calculation of the fraction of the frame covered by negative values, which represents the fraction of the bed covered by fuel particles. Furthermore, the location where fuel particles have been detected throughout the video is also qualitatively determined by this method.

\section{Results and discussion}

A frame from the first camera while the bed (coarse sand) is fluidized at $\mathrm{u}_{0}=0.15 \mathrm{~m} / \mathrm{s}$ is given in Figure 3(a). The same frame after the image has been processed is shown in Figure 3(b), where the fuel particles have been segmented and superimposed on the original frame.

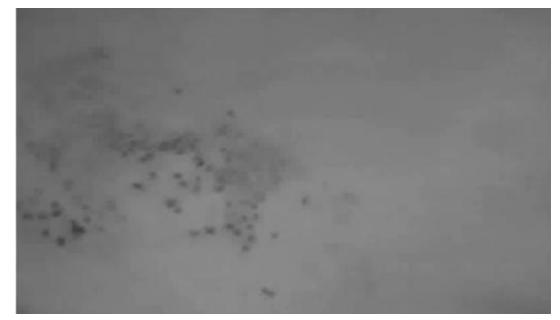

(a)

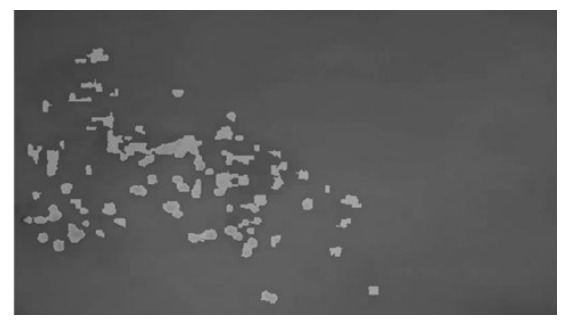

(b)

Figure 3: (a) original frame; (b) frame after data processing.

The zones where the fuel particles have been observed more frequently is given in Figure 4 for the coarse sand case and $u_{0}=0.12 \mathrm{~m} / \mathrm{s}$. The image shows the surface of the bed with shaded areas that represent the regions of the bed where the fuel particles have been detected. The circular shapes are due to condensing gas on the lens. The greyscale is proportional to the fraction of the total frames in which the fuel particles have been detected at a given location. It was observed that in all tests the fuel particles tend to spend longer time in specific regions of the reactor. From the recorded videos it was possible to see that those regions correspond to areas between bubble paths, which is in good agreement with findings by Olsson et al. [14].

The average surface of the bed covered by fuel particles is represented in Figure 5 and Figure 6 as a function of the excess gas velocity $\left(u_{0}-u_{m f}\right)$ and the fluidization number $\left(\mathrm{u}_{\mathrm{o}} / \mathrm{u}_{\mathrm{mf}}\right)$, respectively. Regardless of the fluidization parameter used, increasing fluidization velocity decreases the amount of fuel floating on the surface of the bed. This trend is in agreement with previous research and, thus supports the validity of the experimental method used in this work. 


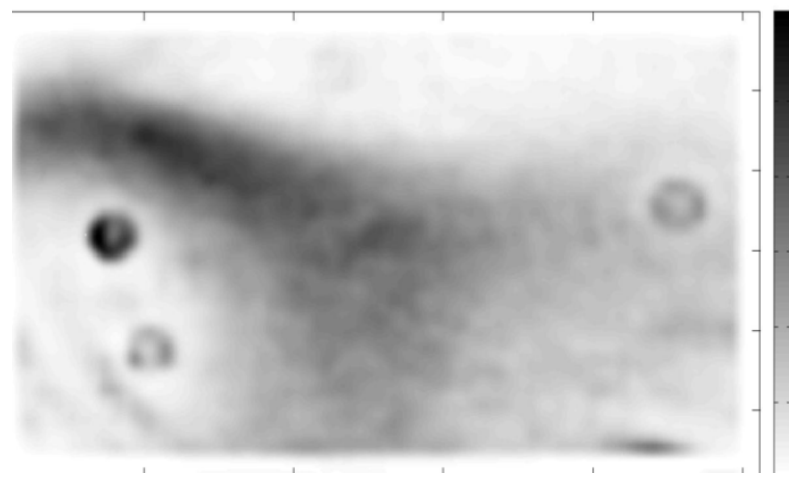

Fuel particles frequently detected

No fuel particles detected

Figure 4: Bed surface with marked areas frequented by fuel particles, according to the greyscale.

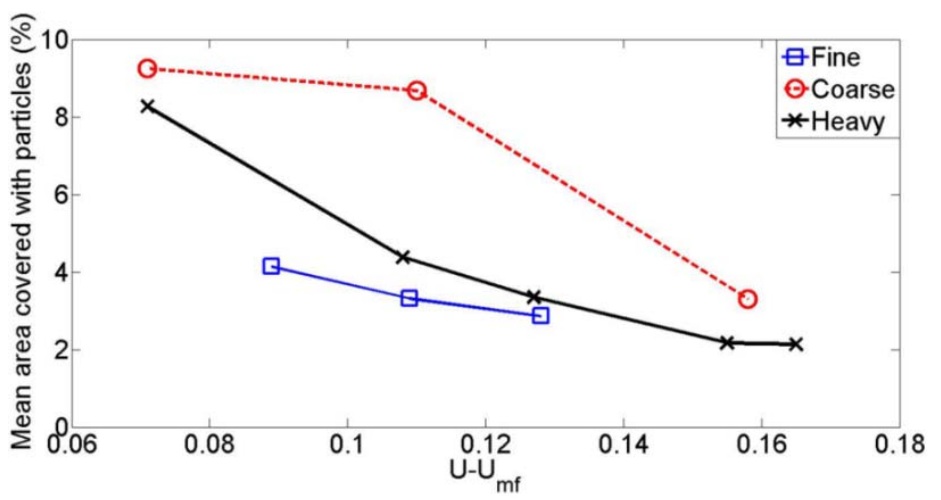

Figure 5: Average area covered with fuel particles as a function of $\left(\mathrm{u}_{\mathrm{o}}-\mathrm{u}_{\mathrm{mf}}\right)$.

From the videos, the bed appears to be fluidized at all levels of fluidization velocities investigated with the fine sand and the heavy sand bed. However, for the case with coarse sand the bed is poorly fluidized at the two lowest fluidization velocities investigated, and particles are clearly floating on the bed surface. This is the explanation for the larger mean area covered with fuel in Figure 5 for the coarse sand case. When the fluidization velocity is increased to a higher level the bed is visually well fluidized and the fuel particles start to mix into the bed, thus the sharp decrease in mean area covered with fuel particles. This suggests that there is a minimum level of fluidization required for the fuel to start mixing with the bed, which corresponds to $3.5 \cdot \mathrm{u}_{\mathrm{mf}}$ for the bed material tested. Operation below such threshold would have major negative impact in units with over-bed fuel feeding, where contact between fuel and bed material particles would be minimized. 


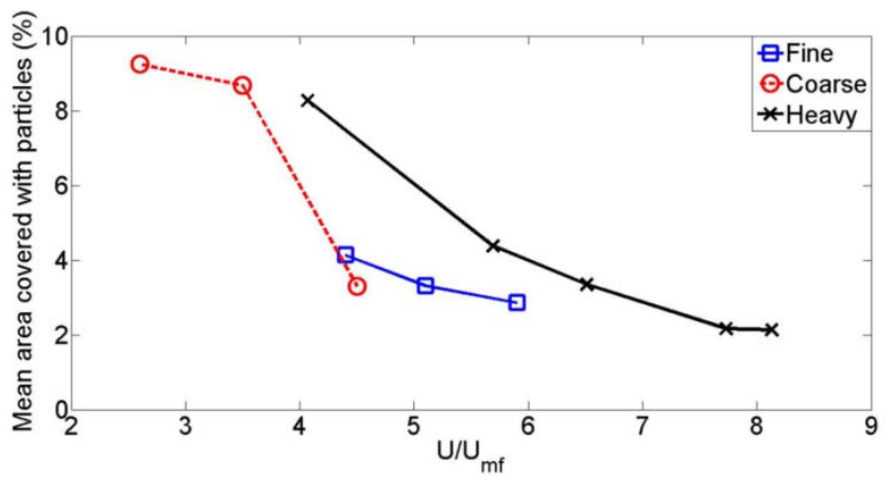

Figure 6: Average area covered with fuel particles as a function of $\mathrm{u}_{\mathrm{o}} / \mathrm{u}_{\mathrm{mf}}$.

On the right hand side of Figure 6 (i.e. heavy sand at approximately $8 \cdot u_{m f}$ ) the positive effect of fluidization velocity on fuel mixing becomes less pronounced. This is in line with previous findings by Zhang et al. for biomass-sand mixtures in cold conditions [3]. The authors found that increasing fluidization number was beneficial up to a certain point. Thereafter, and, depending on the mixing ratio, fluidization velocity did not promote mixing and could even enhance segregation in some cases. The higher the biomass fraction in the biomass-sand mixture, the more significant the fuel segregation was. In the range of fluidization tested in this work it was not possible to see a negative effect of fluidization velocity on vertical mixing. This could be related to the low mixing ratio or to the level of fluidization velocity investigated. Tests at higher fluidization numbers and different mixing ratio would elucidate this issue. Further, as shown in Figure 6, the change of mixing behaviour occurs at $8 \cdot u_{\mathrm{mf}}$, while Zhang et al. observed such change at $4 \cdot u_{\mathrm{mf}}$. This could be explained by the significantly lower density and larger size of the biomass used in their work, which makes the fuel particles more prone to float than in the present investigation.

Although the trends shown in Figures 5 and 6 are similar, the relative position of the curves corresponding to the three bed materials differs. This is a consequence of how the two fluidization parameters are defined. Note that the excess gas velocity $\left(\mathrm{u}_{0}-\mathrm{u}_{\mathrm{mf}}\right)$ is approximately equal to $\mathrm{u}_{0}$, provided that $\mathrm{u}_{\mathrm{mf}}$ is relatively small compared to the fluidization velocities tested. Consequently, for a given steam flow, bed materials with similar fluidization and mixing behaviour appear close to each other in Figure 5. The fluidization number, $\mathrm{u}_{\mathrm{o}} / \mathrm{u}_{\mathrm{mf}}$, on the other hand, represents the level of fluidization and fuel mixing in a more suitable manner when materials of different characteristics are to be compared. As shown in Figure 6, the cases with visually poor fluidization (i.e. coarse sand at low steam flow) are located on the left hand side of the plot, while the visually similar fluidization levels fall close to the same region of the x-axis. For this reason, the effect of the density of the bed material was evaluated using the fluidization number $\mathrm{u}_{\mathrm{o}} / \mathrm{u}_{\mathrm{mf}}$. As expected, the higher density of the bed material enhances the floating tendency of the fuel particles, which results in a larger portion of the surface of the bed covered with fuel in the case of heavy sand. The 
effect of density, however, seems to be more pronounced at lower fluidization velocities, while at higher velocities the bed materials tested behave more similarly. The varying impact of the density ratio between fuel and bed material at high and low fluidization velocity is also found in the context of lateral mixing [15].

\section{Conclusions}

A method to compare the level of segregation of high-volatile fuel particles in a bubbling bed reactor has been developed and applied to a large scale unit. The method is based on digital image analysis of recorded videos, and it enables the quantification of the fraction of the bed surface that is covered by fuel particles. Such parameter was used for qualitative assessment of the level of segregation at different fluidization velocities for three different bed materials. The conclusions derived from the results are as follows:

(1) Fuel segregation was found to be dominant at gas velocities below $3.5 \mathrm{u}_{\mathrm{mf}}$.

(2) Fluidization velocity above $8 \mathrm{u}_{\mathrm{mf}}$ was required to achieve good fuel mixing. Further increase in fluidization velocity did not have a significant effect on vertical mixing any longer.

(3) In the region between those limits, mixing is highly dependent on fluidization velocity regardless the properties of the materials.

(4) The density of the bed material was found to have a larger impact at low fluidization velocities.

(5) The observations support that fuel particles tend to prefer the regions between bubble paths.

\section{Acknowledgement}

This work was supported by Akademiska Hus, Göteborg Energi AB, Valmet Power AB, the Swedish Energy Agency.

\section{References}

[1] Rowe, P.N. and A.W. Nienow, Particle mixing and segregation in gas fluidised beds. A review. Powder Technology, 1976. 15(2): pp. 141-147.

[2] Rowe, P.N., A.W. Nienow and A.J. Agbim, The mechanism by which particles segregate in a gas fluidized bed. Trans.I Chem.Engrs., 1972. 57: pp. 194-199.

[3] Zhang, Y., B. Jin, and W. Zhong, Experimental investigation on mixing and segregation behavior of biomass particle in fluidized bed. Chemical Engineering and Processing: Process Intensification, 2009. 48(3): pp. 745754.

[4] Fiorentino, M., A. Marzocchella, and P. Salatino, Segregation of fuel particles and volatile matter during devolatilization in a fluidized bed 
reactor-II. Experimental. Chemical Engineering Science, 1997. 52(12): pp. 1909-1922.

[5] Bruni, G., Solemine, R., Marzocchella, A., Salatino,P., Yates, J.G, Lettieri, P. and Fiorentino, M. Self-segregation of high-volatile fuel particles during devolatilization in a fluidized bed reactor. Powder Technology, 2002. 128(1): pp. 11-21.

[6] Solimene, R., R. Chirone, and P. Salatino, Characterization of the devolatilization rate of solid fuels in fluidized beds by time-resolved pressure measurements. AIChE Journal, 2012. 58(2): pp. 632-645.

[7] Solimene, R., A. Marzocchella, and P. Salatino, Hydrodynamic interaction between a coarse gas-emitting particle and a gas fluidized bed of finer solids. Powder Technology, 2003. 133(1-3): pp. 79-90.

[8] Leckner, B. and M.R. Golriz, Zhang, W. and Andersson, B-A. Boundary layers-First measurements in the $12 \mathrm{MW} C F B$ research plant at Chalmers university. in 11th International Conference on Fluidized Bed Combustion. 1991. Montreal, Canada: ASME.

[9] Larsson, A., Seeman, M., Neves, D. and Thunman, H., Evaluation of Performance of Industrial-Scale Dual Fluidized Bed Gasifiers Using the Chalmers 2-4-MWth Gasifier. Energy \& Fuels, 2013. 27: pp. 6665-6680.

[10] Thunman, H. and M.C. Seemann. First experiences with the new chalmers gasifier. 2009.

[11] Aerov M.E. and Todes O.M., Hydraulic and Thermal Fundamentals on the Operation of Apparatus with Static and Fluidised Particle Bed, ed. Chimia. 1968: Leningrad.

[12] Bradski, G. and A. Kaehler, Learning OpenCV, ed. M. Loukides. 2011, Sebastopol, CA, United States: O’Reilly.

[13] Nixon, M.S. and A.S. Aguado, Feature Extraction \& Image Processing for Computer Vision. 3 ed. 2012, Oxford, UK: Academic Press.

[14] Olsson, J., D. Pallarès, and F. Johnsson, Lateral fuel dispersion in a largescale bubbling fluidized bed. Chemical Engineering Science. 74(0): pp. 148-159.

[15] Sette, E., D. Pallarès, and F. Johnsson, Experimental quantification of lateral mixing of fuels influid-dynamically down-scaled bubbling beds. Applied Energy, 2014. 136(0): pp. 671-681. 